\title{
Time evaluation of diamagnetic loop and Mirnov oscillations in a major plasma disruption and comparison with a normal shot
}

\author{
R. Sadeghi ${ }^{1} \cdot$ Mahmood Ghoranneviss $^{1} \cdot$ M. K. Salem ${ }^{1}$
}

Received: 21 November 2018 / Accepted: 7 December 2018 / Published online: 21 December 2018

(c) The Author(s) 2018

\begin{abstract}
In this paper, plasma disruption was investigated in IR-T1 tokamak. Moreover, the time evaluation of plasma parameters such as plasma current, $I_{\mathrm{p}}$; loop voltage, $V_{\text {loop}}$; power heating; and safety factor was illustrated. The results of diamagnetic loop and Mirnov oscillations in a major disruption were compared with their results in a normal shot. In addition, plasma disruption in different tokamaks was investigated and the results were compared with the IR-T1 tokamak.
\end{abstract}

Keywords Disruption · Runaway electrons · Mirnov coils · Diamagnetic loop · Tokamak · MHD instability

\section{Introduction}

Among various tools of magnetic confinement, tokamak containing hot plasma is one of the best and developed devices [1]. Tokamaks are divided into two groups: first, the large tokamaks for studying large plasmas such as DIII-D [2] and Tore-Supra [3] with high-cost testing conditions and second, small tokamaks such as STOR-M [4] and ISTTOK [5] which have close relationship with the first group so that most of the experiments are initially carried out by them. One of the applications of tokamaks is in the field of nanoscience. Neverov et al. [6] developed a theoretical model for neutron and X-ray diffraction of carbon nanomaterials and hydrocarbon films deposited inside a vacuum vessel of the tokamak T10. Nechaev and Alexeeva [7] answered to some problems about thermodynamic analysis of hydrogen storage in carbon-based nanomaterials with sp' hybridization which was used in atomic hydrogen adsorption in carbon nanostructures. In addition, Kukushkin et al. [8] stimulated the fine structure of the deposited films deposited in the vacuum vessel of high-temperature plasma such as stellarators and tokamaks. They also created the nanomaterials based on the carbon nanostructures.

IR-T1 tokamak is the small type of tokamak which is used in this experiment. As an important phenomenon,

Mahmood Ghoranneviss

m.ghoranneviss@hotmail.com

1 Department of Physics, Faculty of Sciences, Science and Research Branch, Islamic Azad University, Tehran, Iran tokamak disruptions cause plasma confinement to be destroyed by restriction of current and density which ends to large mechanical stresses. Plasma disruption is caused by strong stochastic magnetic field formed due to nonlinearity excited low-mode number magneto-hydro-dynamics (MHD) modes. The safety factor $(q)$ is very important in determining stability and transport theory [9-13]. The paper is organized as follows: in Sect. 2, time evaluation of plasma parameters and safety factor is summarized; Sect. 3 presents time evaluation of diamagnetic loop and Mirnov oscillations in a major disruption. Moreover, the results are compared with a normal shot; in Sect. 4, we investigate the plasma disruption in the different tokamaks and finally, Sect. 5 includes a summary and conclusions.

\section{The time evaluation of plasma parameters and safety factor}

The IR-T1 tokamak parameters in the disruptive discharges have the density limit $\left(\bar{n}_{\mathrm{e}} \sim 1.5 \times 10^{19} \mathrm{~m}^{-3}\right)$, the approximate plasma current ( $30 \mathrm{KA}$ ) is obtained for $5 \mathrm{~ms}$ approximately and the loop voltage is ( $2 \mathrm{~V})$, major radius is $45 \mathrm{~cm}$, minor radius is $12 / 5 \mathrm{~cm}$ and toroidal field is $1 / 0 \mathrm{~T}$.

Figure 1 shows the position of poloidal array of 12 Mirnov coils [14].

$P_{\text {ohmic }}$ is the rate of input heating power. The ohmic heating power is

$P_{\text {ohmic }}=V_{\mathrm{R}} I_{\mathrm{P}}-\left(\frac{1}{2} \mathrm{LI}_{P}^{2}\right)$ 


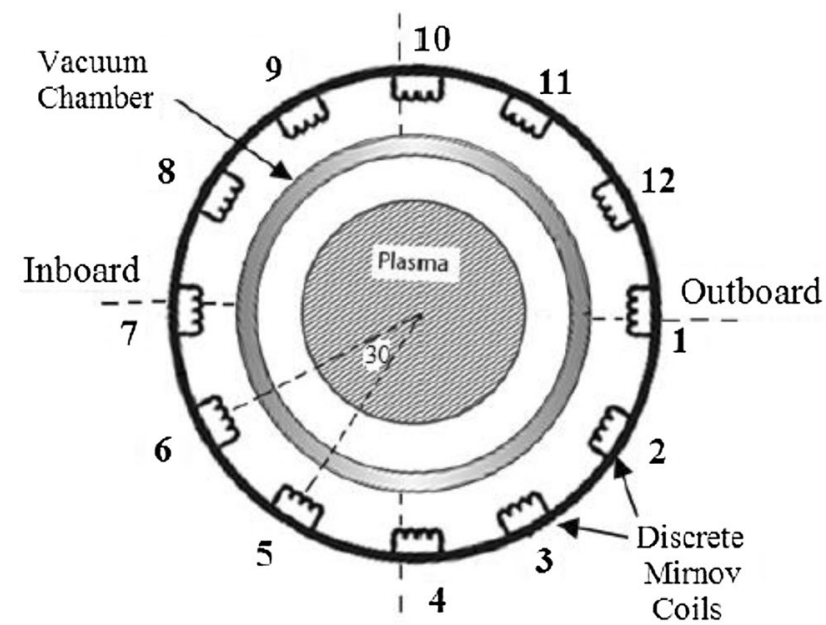

Fig. 1 Position of poloidal array of 12 Mirnov coils

If the plasma is in thermal equilibrium $(\dot{L}=0, \dot{I}=0)$, then we have [15]:

$\mathrm{P}_{\text {ohmic }}=\mathrm{V}_{\mathrm{R}} \mathrm{I}_{\mathrm{P}}$.

Figures 2 and 3 show the experimental signals of a discharge (plasma current, loop voltage and heating power). Figure 4 shows the time evaluation of safety factor. Major disruption is observed in $t=20 \mathrm{~ms}$ that annihilates plasma in $t=15 \mathrm{~ms} . \mathrm{P}_{\text {ohmic }}$ is the rate of input heating power [15].

In this section, we will compare experimental results in the normal and disruption shots obtained in the IR-T1 tokamak.

Plasma evaluations in a normal shot of IR-T1 tokamak,

Plasma evaluations during a disruption of IR-T1 tokamak.

We conclude an increase in MHD oscillations and a loop voltage peak in pre-disruption stage that generate high-energy runaway electrons.

With looking up to above plots in IR-T1 tokamak, we can say that regarding the short discharge duration, plasma disruption will happen very soon and it produces runaway electrons.

In this section, we have the time evaluation of safety factor in IR-T1 tokamak. The safety factor, q, is so called because of the role it plays it determining the stability. If $q=\frac{m}{n}$, where $\mathrm{m}$ and $\mathrm{n}$ are integers, the field line joins up on itself after $\mathrm{m}$ toroidal and $\mathrm{n}$ poloidal rotations round the torus [1].

The safety factor (q) is very important in determining stability and transport theory.

\section{The time evaluation of diamagnetic loop and Mirnov oscillations}

In this section, we study time evaluation of Mirnov oscillations and diamagnetic loop in IR-T1 tokamak in a normal shot and a disruption shot. Figures 5 and 6 show the time

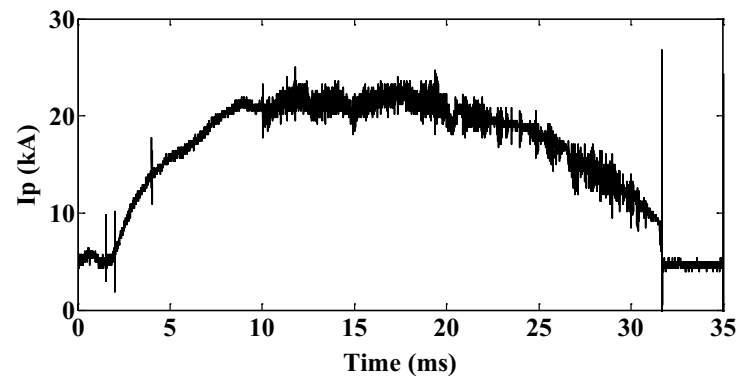

(a)

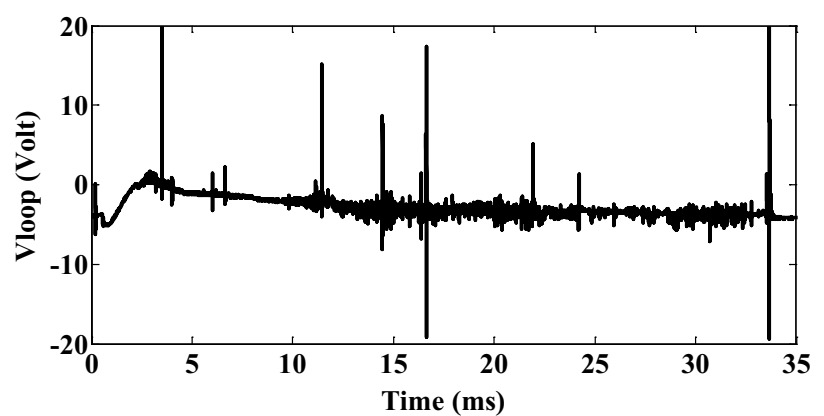

(b)

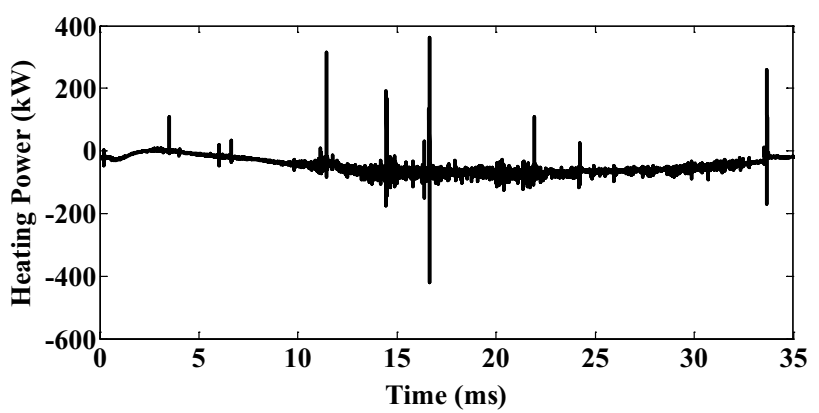

(c)

Fig. 2 a Plasma current, b loop voltage, $\mathbf{c}$ heating power in a normal shot

evaluation of Mirnov oscillations. Figures 7 and 8 show the time evaluation of diamagnetic loop.

We conclude that in pre-disruption stage, increase in MHD oscillations and loop voltage peak causes generation of high-energy runaway electrons.

\section{The plasma disruption in different tokamaks}

In this section, we will investigate the plasma disruption in different tokamaks (JET, TEXTOR, TCV, COMPASS, ASDEX Upgrade, and MAST) and we will compare the results with the IR-T1 tokamak.

In JET, a major disruption occurs in the time $\mathrm{t}=14 / 5$ (s), and $\mathrm{I}=1 / 2 \mathrm{MA}$ and $\mathrm{B}=1 / 2 \mathrm{~T}[16,17]$. 


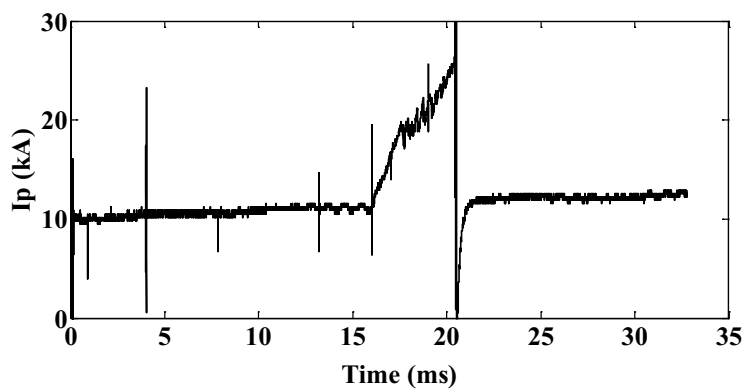

(a)

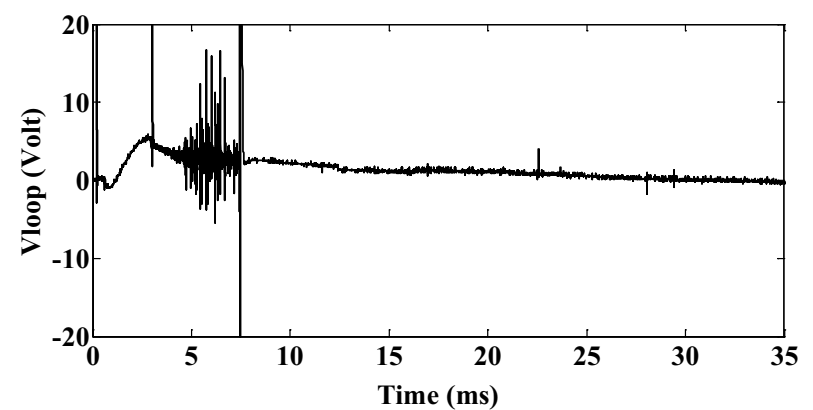

(b)

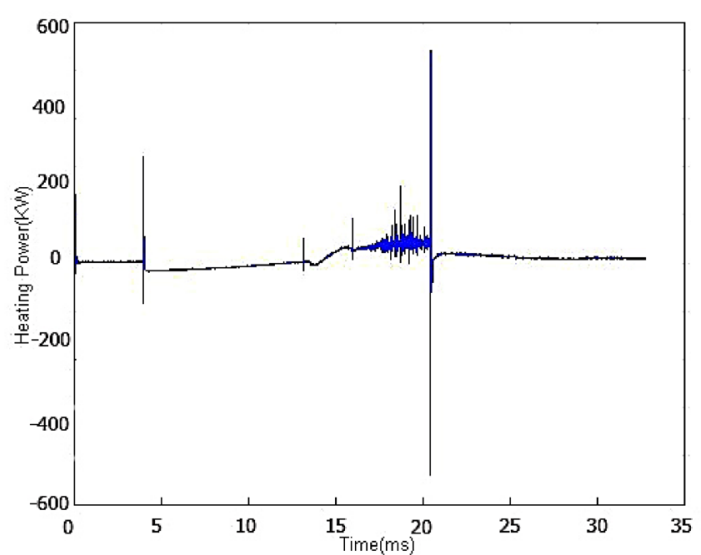

(c)

Fig. 3 a Plasma current, b loop voltage, $\mathbf{c}$ heating power in a disruption shot

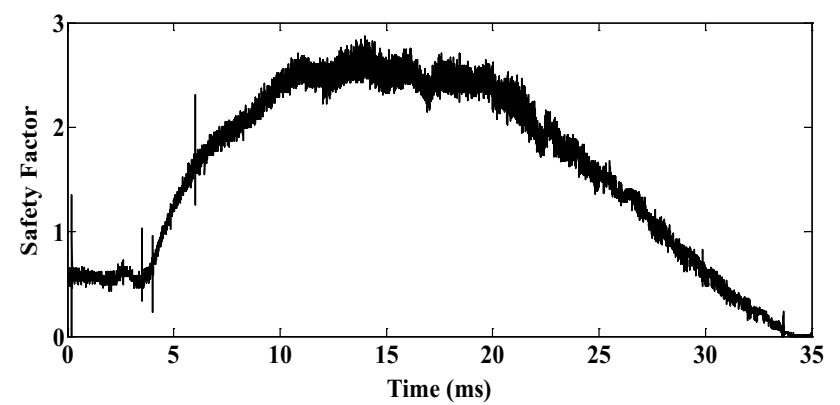

Fig. 4 Time evaluation of safety factor in IR-T1 tokamak

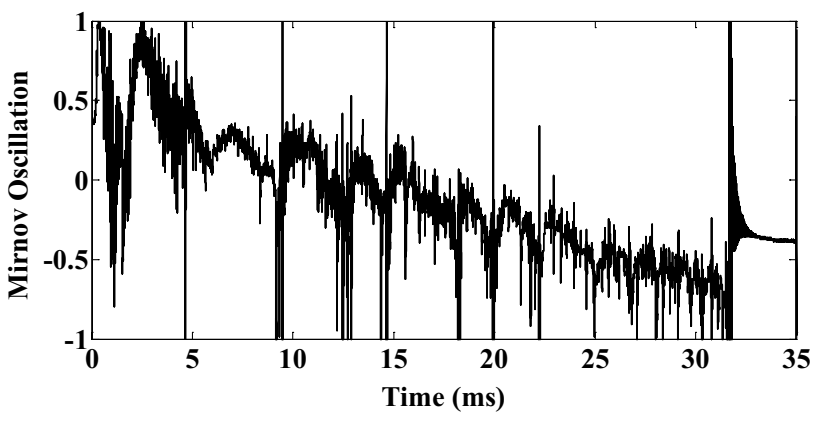

Fig. 5 Time evaluation of Mirnov oscillations in IR-T1 tokamak in a normal shot

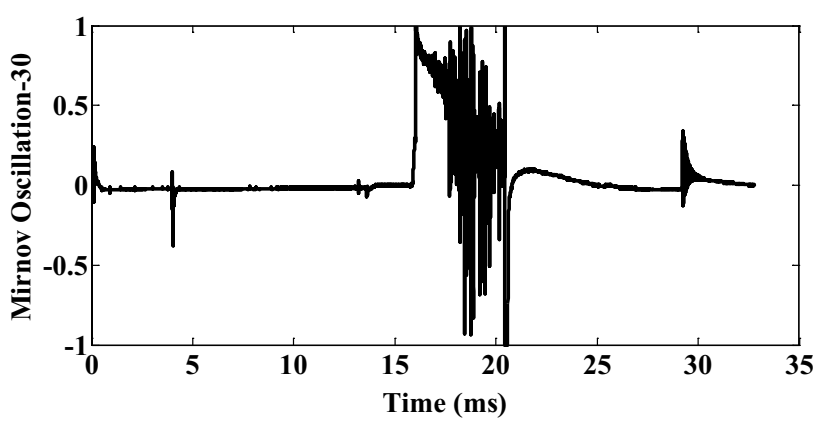

Fig. 6 Time evaluation of Mirnov oscillations in IR-T1 tokamak in a disruption shot

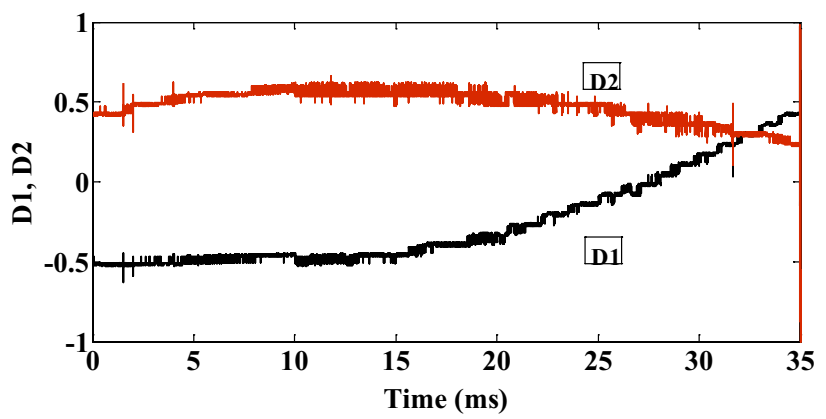

Fig. 7 Time evaluation of diamagnetic loop in IR-T1 tokamak in a normal shot

In TEXTOR, disruption occurs in the time $\mathrm{t}=2 / 01(\mathrm{~s})$, and $\mathrm{I}=350 \mathrm{KA}$ and $\mathrm{B}=2 / 4 \mathrm{~T}[18]$.

In TCV, disruption occurs in the time $t=1 / 5(\mathrm{~s})$, and $\mathrm{I}=200 \mathrm{KA}$ and $\mathrm{B}=1 / 43 \mathrm{~T}$ [19].

In COMPASS, disruption occurs in the time $\mathrm{t}=1 / 12(\mathrm{~s})$, and $\mathrm{I}=80 \mathrm{KA}$ and $\mathrm{B}=1 / 15 \mathrm{~T}$ [19].

In ASDEX Upgrade, disruption occurs in the time $\mathrm{t}=1$ (s), and $\mathrm{I}=0 / 8 \mathrm{MA}$ and $\mathrm{B}=2 / 5 \mathrm{~T}$ [19].

In MAST, disruption occurs in the time $\mathrm{t}=0 / 5(\mathrm{~s})$ and $\mathrm{I}=400 \mathrm{KA}[20]$.

In IR-T1, disruption occurs in the time $\mathrm{t}=20(\mathrm{~ms})$ [15]. 


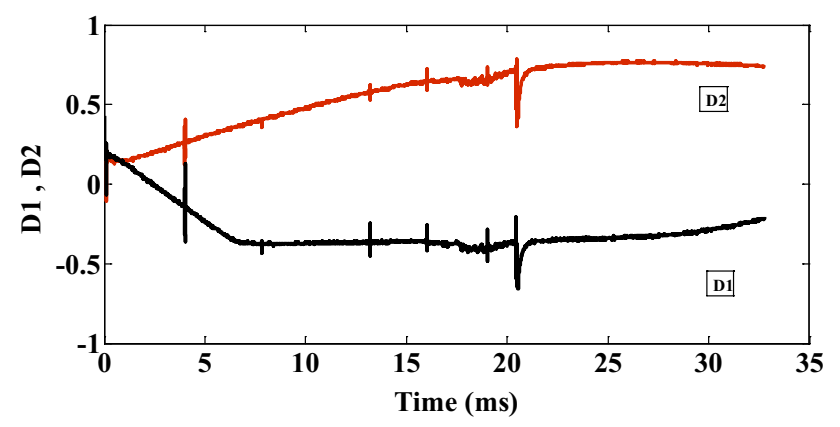

Fig. 8 Time evaluation of diamagnetic loop in IR-T1 tokamak in a disruption shot

We conclude that plasma disruption occurs late in large tokamaks, but early in small tokamaks.

By the way, with looking up to the above plots in IR-T1 tokamak, it is concluded that regarding the short discharge duration, the plasma disruption will happen very soon and it produces runaway electrons, but in large tokamaks like JET, the discharge duration is longer than plasma disruption and production of runaway electrons will happen later.

\section{Conclusions}

Many applications of tokamaks such as nanotechnology, plasma disruption, and plasma heating have been investigated recently. In the present study, some disruption parameters in tokamak were analyzed and measured. We showed the time evaluation of Plasma current, $I_{p}$, loop voltage, $V_{\text {loop }}$ and power heating in a major disruption, and then we compared the results with a normal shot. We also showed the time evaluation of plasma safety factor. We showed an increase in MHD oscillations and loop voltage peak in pre-disruption stage that causes generation of high-energy runaway electrons. We also showed the time evaluation of diamagnetic loop and Mirnov oscillations in a major disruption and then we compared the results with a normal shot. We also investigated the plasma disruption in the different tokamaks and then we compared the results with the IR-T1 tokamak. We conclude that plasma disruption occurs late in large tokamaks, but early in small tokamaks.

\section{Compliance with ethical standards}

Conflict of interest The authors report no conflict of interests. The authors alone are responsible for the content and writing of the paper.

Open Access This article is distributed under the terms of the Creative Commons Attribution 4.0 International License (http://creativeco mmons.org/licenses/by/4.0/), which permits unrestricted use, distribution, and reproduction in any medium, provided you give appropriate credit to the original author(s) and the source, provide a link to the Creative Commons license, and indicate if changes were made.

\section{References}

1. Wesson, J.A.: Disruptions in JET. Nucl. Fusion 29, 641 (1989)

2. Burrell, K.H., Gohil, P., Groebner, R.J., Kaplan, D.H., Robinson, J.I., Solomon, W.M.: Improved charge-coupled device detectors for high-speed, charge exchange spectroscopy studies on the DIIID tokamak. Rev. Sci. Instrum. 75, 3455 (2004)

3. Hennequin, P., Honoré, C., Truc, A., Quéméneur, A., FenziBonizec, C., Bourdelle, C., Garbet, X., Hoang, G.T.: Fluctuation spectra and velocity profile from Doppler backscattering on Tore Supra. Nucl. Fusion 46, S771 (2006)

4. Onchi, T., Liu, Y., Dreval, M., McColl, D., Elgriw, S., Liu, D., Asai, T., Xiao, C., Hirose, A.: Effects of compact torus injection on toroidal flow in the STOR-M tokamak. Plasma Phys. Controlled Fusion 55, 035003 (2013)

5. Gomes, R.B., Varandas, C.A.F., Cabral, J.A.C., Sokolova, E., Cortes, S.R.: High dispersion spectrometer for time resolved Doppler measurements of impurity lines emitted during ISTTOK tokamak discharges. Rev. Sci. Instrum. 74, 2071 (2003)

6. Neverov, V.S., Voloshinov, V.V., Kukushkin, A.B., Tarasov, A.S.: Influence of molecular clustering on the interpretation of diffractograms of hydrocarbon films from tokamak T-10. Phys. At. Nucl. 78, $1112(2015)$

7. Nechaev, Y.S., Alexeeva, O.K.: On the nature, capability and reversibility of hydrogen storage in novel carbon nanomaterials for mobile power units. Int. J. Hydrogen Energy 28, 1433 (2003)

8. Kukushkin, A.S., Pacher, H.D., Loarte, A., Komarov, V., Kotov, V., Merola, M., Pacher, G.W., Reiter, D.: Analysis of performance of the optimized divertor in ITER. Nucl. Fusion 49, 075008 (2009)

9. Horton, W.: Drift waves and transport. Rev. Mod. Phys. 71, 735 (1999)

10. Schuller, F.: Disruptions in tokamaks. Plasma Phys. Control Fusion 37, A135 (1995)

11. Ghanbari, M.R., Ghoranneviss, M., Mohammadi, S., et al.: Runaway electron generation decrease during a major disrption by limiter biasing in tokamaks. Radiat. Eff. Defects Solids 168, 664 (2013)

12. Mukhovatov, V.S., Shafranov, V.D.: Plasma equilibrium in a Tokamak. Nucl. Fusion 11, 605 (1971)

13. Zakharov, L.E., Shafranov, V.D.: Equilibrium of a toroidal plasma with noncircular cross section. Sov. Phys. Tech. Phys. 18, 151 (1973)

14. Goodarzi, Z., Ghoranneviss, M., Salarelehi, A.: Investigation of plasma MHD Activity Using FFT Analysis of Mirnov Oscillations. J Fusion Energy 32, 103 (2013)

15. Saadat, S., Salem, M.K., Ghoranneviss, M., Khorshid, P.: Mode Analysis with Autocorrelation Method (Single Time Series) in Tokamak. J. Fusion Energy 20, 371 (2010)

16. Baker, D.R., Wade, M.R., Petty, C.C., et al.: Particle transport phenomena in the DIII-D tokamak. Nucl. Fusion 40, 1003 (2000)

17. Martin Solis, J.R., Alvarez, D., Sanchez, R.: Momentum-Space structure of relativistic runaway electrons. Phys. Plasmas 5, 2370 (1998)

18. Kim, J.S., Edgell, D.H., Greene, J.M., Strait, E.J., Chance, M.S.: MHD mode identification of tokamak plasmas from Mirnov signals. Control Fusion 41, 1399 (1999) 
19. Constantinescu, D., Dumbrags, O., Igochine, V., Weyssow, B.: On the accuracy of some mapping teqniques used to study the magnetic field dynamics in tokamaks. Nucl. Fusion 48, 024017 (2008)

20. Kadomstev, B.B.: Plasma transport in Tokamaks. Nucl. Fusion 31, 2201 (1991)

Publisher's Note Springer Nature remains neutral with regard to jurisdictional claims in published maps and institutional affiliations. 